\title{
Modelling the Late-time Hubble Space Telescope Imaging of the Outburst of the Recurrent Nova RS Ophiuchi (2006)
}

\author{
Valério A. R. M. Ribeiro, ${ }^{1} \dagger$ Michael F. Bode, ${ }^{2}$ and Robert Williams ${ }^{3}$ \\ ${ }^{1}$ Astrophysics, Cosmology and Gravity Centre, Department of Astronomy, \\ University of Cape Town, Private Bag X3, Rondebosch, 7701, South Africa \\ email: vribeiro@ast.uct.ac.za \\ ${ }^{2}$ Astrophysics Research Institute, Liverpool John Moores University, \\ Egerton Wharf, Birkenhead, CH41 1LD, UK \\ ${ }^{3}$ Space Telescope Institute, 3700 San Martin Drive Baltimore, MD 21218 USA
}

\begin{abstract}
The nebular remnant of RS Ophiuchi was modelled using combined HST/ACS imaging and ground-based spectroscopy on day 155 after outburst as a two component bipolar expansion with a low velocity innermost hour-glass over density and a more extended high velocity dumbbell structure. The model was evolved to a much later date, day 455 after outburst, when second epoch HST images were secured. However, due to the lack of simultaneous ground-based spectroscopy the evolved model was much harder to constrain. One suggestion put forward was that the dumbbell structure expanded linearly while the inner hour-glass showed signs of deceleration. Archival data in the form of ground-based spectroscopy on day 415 were subsequently obtained. These new data suggest indeed that a non-linear expansion of the system occurred.
\end{abstract}

Keywords. binaries: symbiotic, circumstellar matter, line: profiles, novae, cataclysmic variables, stars: individual (RS Ophiuchi)

\section{Introduction}

RS Ophiuchi is a member of the small group of objects known as symbiotic recurrent novae, with seven confirmed recorded outbursts. The system comprises a white dwarf, probably close to the Chandrasekhar limit (e.g. Brandi et al. 2009) and a red-giant secondary of spectral type estimated around M0/2 III (e.g. Anupama \& Mikołajewska 1999). Oph's latest outburst, first detected on 2006 February 12.83 (Narumi et al. 2006), reached peak on 2006 February 12.94 (Hounsell et al. 2010) and was observed from the hard X-ray to the radio (for a recent review see Ribeiro 2011, and references therein).

The system was first resolved as a partial ring of non-thermal radio emission using Very Long Baseline Interferometry on day 13.8 after outburst. As the system evolved, a bipolar structure emerged. The asymmetry was suggested to be due to absorption in the overlying red-giant wind (O'Brien et al. 2006, 2008). Very Long Baseline Array imaging between days $34-51$ after outburst showed a central thermally dominated source linked to what appeared to be a collimated non-thermal outflow (Sokoloski et al. 2008). Taylor et al. (1989) also interpreted their observations from day 77 after the 1985 outburst as a central thermal source and expanding non-thermal lobes.

Ribeiro et al. (2009) used combined Hubble Space Telescope (HST) imaging (using the ACS/HRC F502N filter) and ground-based spectroscopic observations on day 155 after out burst to compare with a model of RS Oph using a morpho-kinematical code

\section{$\dagger$ South African Square Kilometre Array Fellow.}


to retrieve the underlying $3 \mathrm{D}$ structure. They modelled the system as having a bipolar morphology composed of a high velocity outer dumbbell and a low velocity innermost hour glass higher density structure. The innermost hour glass structure replicated well the observed low velocity line width. Ribeiro et al. (2009) were also able to show that the asymmetry on the observed image was due to the finite width and offset of the central wavelength of the F502N filter. They derived the inclination of the system as $39_{-9}^{+1}$ degrees and maximum expansion velocity of $5000_{-100}^{+1500} \mathrm{~km} \mathrm{~s}^{-1}$. One key question that was left open was what happened to the morphology of the system as it evolved from day 155 to later epochs after outburst. Was it a linear expansion of both components or was there something else? This short contribution aims to shed light on this question, following archival data search, by modelling the additional HST imaging from day 449 and ground based spectroscopy (around the same day).

\section{Methods}

RS Oph was imaged with HST under the Directors Discretionary programs GO/DD11075 on 2007 May 7 (day 449 after outburst) with the Planetary Camera CCD of the Wide Field Planetary Camera 2 (WFPC2), with a scale of $0.046^{\prime \prime} \mathrm{pixel}^{-1}$. The HST data reduction steps have already been described in Ribeiro et al. (2009) and will not be detailed here. An archival search of the Isaac Newton Group database proved fruitful in finding a spectroscopic observation of RS Oph on 2007 April 03 (day 415 after outburst) with the $4.2 \mathrm{~m}$ William Herschel Telescope. The observation used the Intermediate Dispersion Spectrograph and Imaging System, using granting R300B with a dispersion of $0.86 \AA /$ pix. The spectra were reduced using standard Image Reduction and Analysis Facilitył procedures. However, no flux calibration was attempted as at this stage we are only interested in the line profile.

\section{Results}

We identified the lines in the region around $5007 \AA$ which at day 155 after outburst was dominated by the [O III] $5007 \AA$ line, with a few lines of Fe II and He I $5018 \AA$ which were identified as originating from the pre-existing red giant wind. However, at the time of the spectroscopic observations on day 415 the lines are much broader and there is no evidence for [O III] 5007 . The broad lines are identified as arising from Nitrogen and Helium (Figure 1). We centre the velocity on N II $5007 \AA$. Also shown in Figure 1 is the $H S T$ WFPC F502N filter profile. This means that in the observed emission on the HST image at day 415 is dominated by emission lines of Nitrogen and Helium.

As a first approximation, we applied the morpho-kinematical code SHAPE 9 (Steffen et al. 2011) using the morphologies from the day 449 results of Ribeiro et al. (2009). Two alternatives were considered: i) A linear expansion of both the inner and outer components, keeping inclination and axial ratios the same, which would produce the same line profile as for day 155 ; ii) a model where the outer component was allowed to expand linearly, while the inner component was kept the same physical size. The results are shown in Figure 2. What is clearly evident is that a model with a linear expansion of both components does not fit the observed line profile. To replicate the line profile,

$\dagger$ http://casu.ast.cam.ac.uk/casuadc/archives/ingarch

$\ddagger$ IRAF is distributed by the National Optical Astronomical Observatories, which is operated by the Associated Universities for Research in Astronomy, Inc., under contract to the National Science Foundation.

I Available at: http://bufadora.astrosen.unam.mx/shape/ 


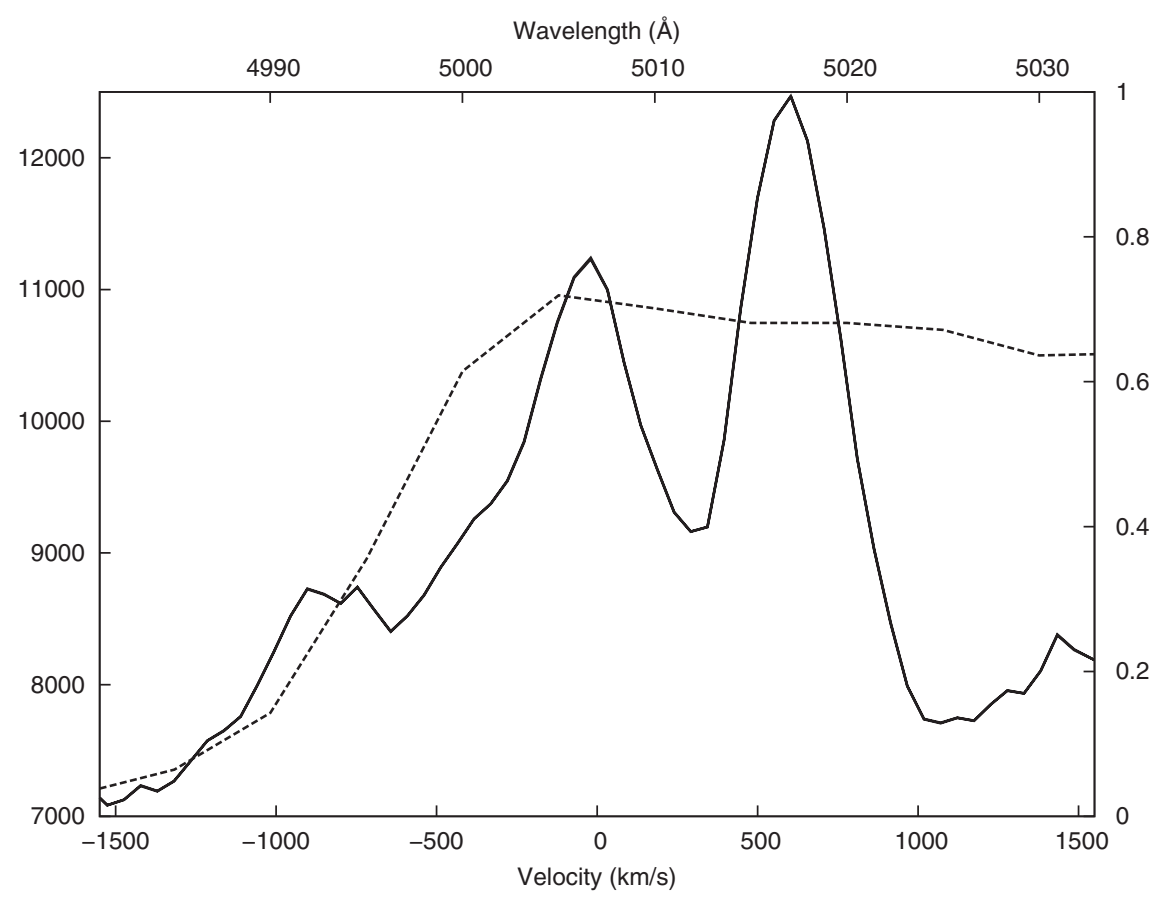

Figure 1. Observed spectrum at day 415 after outburst (solid line) along with the HST F502N filter profile (dashed line).

with model (ii), once again it was required to have an over density in the innermost component.

\section{Discussion and Conclusions}

The change of emission line with which the models of the structure of RS Oph were originally compared appears to make no difference in the overall derived morphology of the system. The relative weakness of forbidden lines in the late-time spectra suggests emission may now be arising predominantly from dense clumps as the general trend is obviously for the density to drop with time. The morphology was well modelled with an outer dumbbell and an inner hour glass structure as on day 155. The inner structure unsurprisingly has a velocity less than the outer component and is much denser. This was required to replicate the observed low velocities seen in the lines. However, the innermost component appears to suffer significant deceleration compared with the outer component. We are now working on identifying all the lines in the archival spectra. Using a wide time span of spectra we can also track the evolution of the outburst (Ribeiro et al., in preparation).

\section{Acknowledgements}

This paper makes use of data obtained from the Isaac Newton Group Archive which is maintained as part of the CASU Astronomical Data Centre at the Institute of Astronomy, Cambridge. VARMR would like to acknowledge financial support from URC, RAS and the IAU through various grants. The South African SKA Project is acknowledged for funding the postdoctoral fellowship position at the University of Cape Town. 


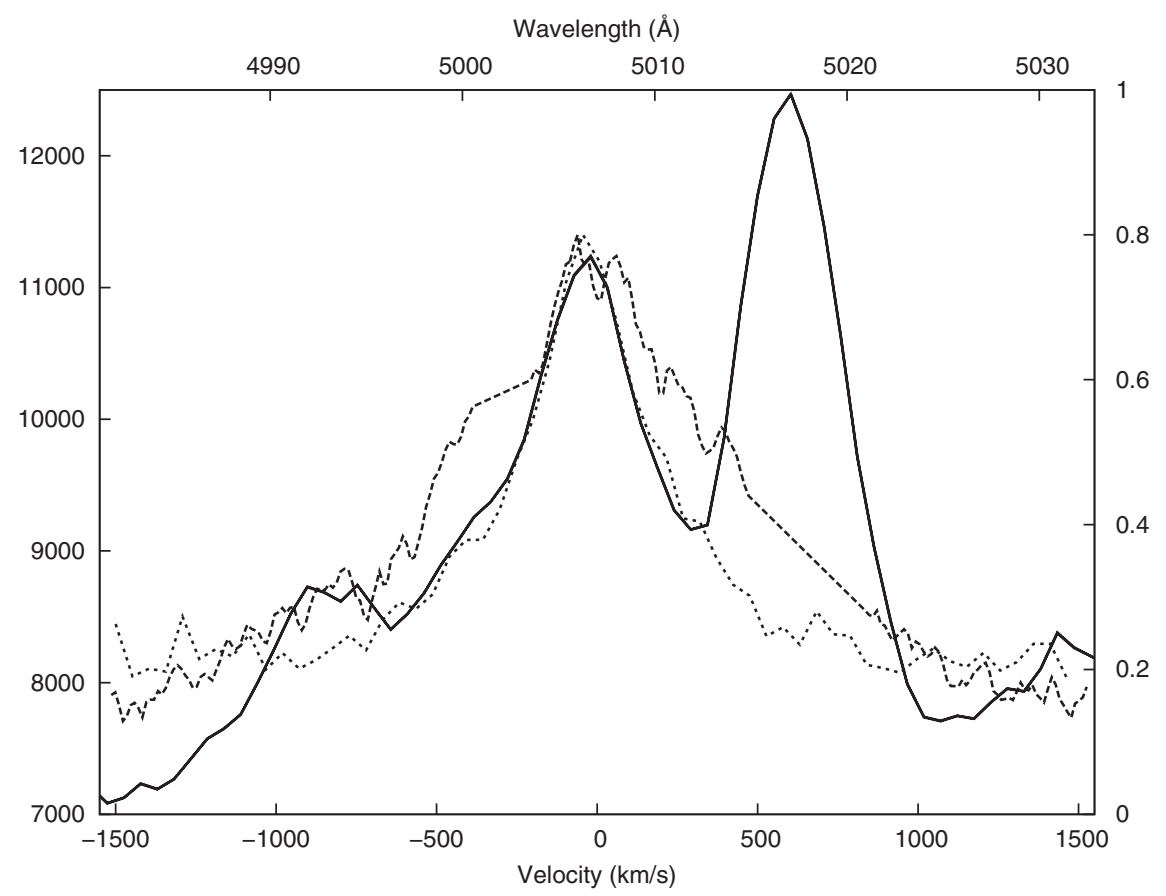

Figure 2. The observed line profile at day 415 after outburst (solid line) along with model line profiles of the different morphologies; the linear expansion of all components (dashed line) and the linear expansion of the outer component while the inner component was kept the same size (dotted line). Note that detailed structure is the line profiles is an artifact due to the finite resolution of the modelling grid.

\section{References}

Anupama, G. C. \& Mikołajewska, J. 1999, A\&A A, 344, 177

Brandi, E., Quiroga, C., Mikołajewska, J., Ferrer, O. E., \& García, L. G. 2009, AE\&A, 497, 815

Hounsell, R., Bode, M. F., Hick, P. P. et al. 2010, ApJ, 724, 480

Narumi, H., Hirosawa, K., Kanai, K. et al. 2006, IAU Circ., 8671

O'Brien, T. J., Beswick, R. J., Bode, M. F. et al. 2008, in Astronomical Society of the Pacific Conference Series, ed. A. Evans, M. F. Bode, T. J. OBrien, \& M. J. Darnley, 401, 239

O'Brien, T. J., Bode, M. F., Porcas, R. W. et al. 2006, Nature, 442, 279

Ribeiro, V. A. R. M. 2011, in The Golden Age of Cataclysmic Variables and Related Objects, F. Giovannelli 85 L. Sabau-Graziati (eds.), Mem. SAIt. 83 N.2 (in press), arXiv, 1110.6330

Ribeiro, V. A. R. M., Bode, M. F., Darnley, M. J. et al. 2009, ApJ, 703, 1955

Sokoloski, J. L., Rupen, M. P., \& Mioduszewski, A. J. 2008, ApJL, 685, L137

Steffen, W., Koning, N., Wenger, S., Morisset, C., \& Magnor, M. 2011, IEEE Transactions on Visualization and Computer Graphics, 17, 454

Taylor, A. R., Davis, R. J., Porcas, R. W., \& Bode, M. F. 1989, MNRAS, 237, 81 